\section{Liquor-IgA, oligoklonal}

T. O. Kleine

Institut für Laboratoriumsmedizin und Pathobiochemie, Molekulare Diagnostik Standort Marburg Referenzlabor für Liquordiagnostik, UKGM Universitätsklinikum Gießen und Marburg, Marburg, Deutschland

Synonym(e) Oligoklonale Immunglobulin-IgA-Banden im Liquor cerebrospinalis

Englischer Begriff oligoclonal IgA bands in CSF

Definition Oligoklonale IgA-Banden (IgA-OB) in CSF, mittels $\triangleright$ isoelektrische Fokussierung (IEF) und immunspezifischer Bandendetektion von monomerem und dimerem IgA in Agarosegel mit Ausschlussgrenze von 2 IgA-OB dargestellt, sind Kenngröße von subakut/chronischen Entzündungsprozessen im Zentralnervensystem (ZNS) mit geringerer Häufigkeit als IgG-OB ( $\triangleright$ Liquor-IgG, oligoklonal).

Struktur $\triangleright$ Immunglobulin A.

Molmasse Monomer 160 kDa; Dimer 335 kDa.

Untersuchungsmaterial - Entnahmebedingungen Ventrikel-, Subokzipital-(SOP-), Lumbal-Liquor.
Probenstabilität Sofort entzellt, aliquotiert in sterilen Plastikröhrchen mit Schraubverschluss und stabil bei $-80{ }^{\circ} \mathrm{C}$ bis zu 1 Jahr gelagert.

Analytik IEF in Agarosegel ( $\triangleright$ Liquor-Agarosegelelektrophorese, $>$ Liquor-Isoelektrische Fokussierung).

Diagnostische Wertigkeit Detektion von IgA-OB mittels IEF mit spezifischer Immundetektion ist verfahrensabhängig: IgA-OB-Test mit monomer/dimerer IgA-Detektion in nativer CSF ist 4-mal weniger häufig positiv als mit Reiber-Diagramm von 1987, bei monomerer IgA-Detektion in reduziertem CSF seltener positiv (59 \% der Fälle) als mit ReiberFelgenhauer-Formel von 1987 (83\%). Falsch-negative Werte bei IgA-Index durch Unterbestimmung von dimerem IgA ( $\triangleright$ Liquor-Immunnephelometrie, partikelverstärkt, QIgA). Fazit: falsch-positive Ergebnisse bei Berechnung von intrathekaler IgA-Produktion durch Unterkompensation von Plasma-IgA in CSF ( $\triangleright$ Immunglobulinbestimmung, intrathekal empirisch [Reiber-Schema]).

\section{Literatur}

Sindic CJM, Monteyne P, Bigaignon G et al (1994) Polyclonal and oligoclonal IgA synthesis in the cerebrospinal fluid of neurological patients: an immunoaffinity-mediated capillary blot study. J Neuroimmunol 49:109-114 